PDFlib PLOP: PDF Linearization, Optimization, Protection

Page inserted by evaluation version www.pdflib.com - sales@pdflib.com 


\section{Health-Related Quality of Life Issues in Women With Polycystic Ovary Syndrome}

Judy Griffin McCook, Nancy E. Reame, and Samuel S. Thatcher

Objective: To evaluate the influence of obesity, fertility status, and androgenism scores on healthrelated quality of life in women with polycystic ovary syndrome (PCOS).

Design: Cross-sectional, correlational.

Setting: Private reproductive endocrinology practice in two southeast U.S. cities.

Participants: Convenience sample of 128 women with PCOS, half of whom were attempting to conceive in addition to being treated for PCOS. Most were White $(97 \%)$, married $(78 \%)$, with a mean age of 30.4 years $(S D \pm 5.5)$.

Main Outcome Measures: The Health-Related Quality of Life Questionnaire (PCOSQ) for women with polycystic ovary syndrome. A laboratory panel and clinical measures, including body mass index, waist-to-hip ratio, and degree of hirsutism.

Results: The most common health-related quality of life concern reported by women with PCOS was weight, followed in descending order by menstrual problems, infertility, emotions, and body hair.

Conclusions: The psychological implications of PCOS are easily underestimated and have been largely ignored. Nursing has a pivotal role in recognizing these concerns and implementing therapy to improve quality of life in women with PCOS. JOGNN, 34, 1220; 2005. DOI: 10.1177/0884217504272945

Keywords: Emotions-PCOS-Polycystic ovaries-Psychological-Quality of life

Accepted: January 2004

Polycystic ovary syndrome (PCOS), formerly referred to as Stein-Leventhal syndrome or polycys- tic ovarian disease, is a complex hormonal disturbance often associated with infertility and myriad health issues. Although there is a typical clinical triad of obesity, skin and hair concerns, and menstrual problems, not all women will share all of the manifestations of PCOS. The varied presentation complicates both diagnosis and treatment of the syndrome. PCOS is believed to be primarily genetic in origin, and presently no cure is available. Treatment is aimed at symptomatic improvement. Women with PCOS symptoms visit on average 4.5 physicians before a diagnosis of PCOS is reached (Cecchine, 2001). Women consult gynecologists regarding menstrual cycle disorders; primary care providers and internists for hyperlipidemia, insulin resistance, and possibly hypertension; dermatologists for hair and skin concerns; and psychiatric providers for treatment of depression and body image disturbances. Each provider in turn treats a few isolated symptoms without recognizing the underlying etiology of this multidimensional syndrome. It is this broad spectrum of symptoms that makes provision of holistic care essential.

Estimated to impact between 5 and 10 million women in the United States alone, PCOS cuts across

COS is a common female endocrine disturbance and the leading cause of female infertility. 
the boundaries of all medical disciplines. The exact incidence of PCOS is unclear because of variances in diagnostic criteria and a failure of experts in the field to agree on a definition. As many as $30 \%$ of all women may have polycystic ovaries on transvaginal ultrasound exam $(>10$ follicular cysts $\leq 10 \mathrm{~mm}$ ). More than half of these women have a second feature of PCOS, such as laboratory abnormalities or clinical findings. About $10 \%$ of women will have all three criteria suggestive of PCOS: altered laboratory parameters, polycystic ovaries on ultrasound, and positive clinical findings (Thatcher, 2000).

Although there is a long-standing interest in PCOS, with more than 5,000 scientific publications, the primary focus of investigation has been on the pathophysiology and treatment of PCOS. Despite the separate and significant psychological impact of all components of the syndrome (infertility, diabetes, and cardiovascular disease) the academic community has largely ignored the collective emotional consequences of PCOS. Only recently has the complexity of the symptomatology of PCOS begun to be investigated in a comprehensive manner to address the psychosocial implications.

The notion of "health-related quality of life" has been described by Colwell, Mathias, Pasta, Henning, and Steege (1998) as a multidimensional concept comprising the physical, psychological, and social components of a designated condition or its treatment. In a recent review, Jones, Kennedy, and Jenkinson (2002) published a summary of health-related quality of life instruments used in gynecologic investigations. They concluded that common benign gynecologic conditions including PCOS were "major sources of psychologic morbidity, and typically lead to an overall diminished [quality of life]" (Jones et al., 2002, p. 508). Evidence is emerging in support of including psychosocial screening tools as a means of providing comprehensive care to women with chronic gynecologic disorders (Colwell et al., 1998; Jones et al., 2002).

In 1998, Cronin et al. (1998) published the HealthRelated Quality of Life Questionnaire for Women with PCOS (PCOSQ), which was the first instrument designed to measure specific PCOS disease-related psychological dysfunction. The authors' intent was to develop a clinically useful instrument that identified emotional, physical, and social problems that PCOS women experienced as a result of having their condition, and to grade the severity of how the symptoms impacted their daily life (Cronin et al., 1998).

Clinicians and researchers co-developed the PCOSQ instrument (Cronin et al., 1998) in three phases. In phase 1 (item selection), a review of the literature, along with feedback from both health professionals and 10 PCOS patients, yielded 182 items that were categorized into eight domains. For phase 2 (item reduction), 100 women with PCOS from three different clinical sites were interviewed and asked to rank the importance and impact of each item. The items were examined for frequency (proportion of women reporting the symptom), importance (mean importance score attached to that item), and impact (frequency multiplied by mean importance) (Cronin et al., 1998). After item reduction, the final version of the PCOSQ instrument had 26 items from five specific domains (subscales) identified as being important to women with PCOS. These were menstrual problems, infertility, weight, body hair, and emotions. This article reports the results of a further test of this questionnaire in a population of PCOS women stratified by body weight, androgenism, and fertility status.

\section{Methods}

\section{Design}

This was a cross-sectional, correlational study of women with PCOS who were patients of a private reproductive endocrinology practice. Prior to the conduct of the study, permission was obtained from Human Subjects Review Committees at both the University of Michigan and East Tennessee State University. The reproductive medicine practice where the women were recruited was located in two small cities in southern Appalachia. Women who had been previously diagnosed with PCOS and were scheduled for a routine follow-up appointment were invited to participate.

As part of a larger study of factors affecting the psychosocial health and well-being of women with PCOS (McCook, 2002), a one-time self-administered health survey containing eight questionnaires was administered during the 1st week of the participant's menstrual cycle. Results from one instrument, the PCOSQ (Cronin et al., 1998), are presented here and examined for the influence of obesity, fertility status, and androgenism. In addition, laboratory and clinical evaluation of hyperandrogenism, as well as information regarding the participant's reproductive history and body mass index, were collected.

\section{Sample}

To be included, participants had to be at least 18 years old; able to read, write, and understand English; and have no chronic disease outside PCOS. Diagnosis of PCOS was made by the presence of at least two of the three following criteria:

1. Laboratory abnormalities (at least one of the three must be abnormal):

a. Reverse in the ratio of luteinizing hormone (LH) to follicle stimulating hormone (FSH)

b. Elevated free androgen index (FAI)

c. Elevated fasting insulin levels $(\geq 16 \mu \mathrm{IU} / \mathrm{ml})$ or stimulated insulin after 1-hour 75-g glucose load of $\geq 100 \mathrm{mg} / \mathrm{dL}$ ) 
2. Transvaginal ultrasound ( $a$ and/or $b$ ):

a. Ovaries with $\geq 10$ cystic structures $\leq 10 \mathrm{~mm}$ in diameter

b. Ovarian volume in excess of $8 \mathrm{ml}$

3. Clinical findings (at least one of the three criteria):

a. Obesity (body mass index $[\mathrm{BMI}]>30.0$ ) or elevated waist-to-hip ratio (WHR) greater than 0.85

b. Skin and hair concerns including recurrent acne and associated scarring, seborrhea, balding, acanthosis nigricans, or hirsutism

c. Menstrual disturbance: amenorrhea, oligomenorrhea, or anovulation

Participants were excluded for abnormally elevated thyroid stimulation hormone (TSH), prolactin, and/or FSH values. All hormonal evaluations were performed in the early follicular phase, and participants with elevated estradiol $\left(E_{2}\right)$ were excluded. With this selection criteria, 128 of the 158 women screened provided data for analysis.

\section{Procedures}

After the study was explained and written consent to participate was obtained from each participant, fasting labs were drawn. Participants were requested to complete a detailed health survey, including the PCOSQ (Cronin et al., 1998), while they were waiting for scheduled consultations with their physician and nutritionist.

\section{Laboratory}

Serum was obtained from each participant after an overnight 8-hour fast during the early follicular phase of the menstrual cycle to obtain measures of $\mathrm{E}_{2}$, progesterone $\left(\mathrm{P}_{4}\right), \mathrm{LH}, \mathrm{FSH}$, prolactin, TSH, total testosterone (TT), and sex hormone binding globulin (SHBG). TT and SHBG were used to calculate the FAI, which is presumed to be a useful indicator of abnormal androgen status (Vankrieken, 1997). The calculation of FAI for this study was derived using the conversion factor for Immulite TT, which was $\mathrm{ng} / \mathrm{mL} \times 3.467=\mathrm{nmol} / \mathrm{L}$ and was calculated as follows: $\mathrm{FAI}=(\mathrm{TT}$ in $\mathrm{nmol} / \mathrm{L} \times 3.467) / \mathrm{SHBG}$ in $\mathrm{nmol} / \mathrm{L}$ (Vankrieken, 1997).

All hormone analyses were completed on site at the Center for Applied Reproductive Science Central lab in Johnson City, TN. Diagnostic Products Corporation's (Los Angeles, CA) automated, nonisotopic immunoassay platform, the IMMULITE, was the instrument used for the analysis of all reproductive hormone assays. All analyses were completed using commercial assay kits from Diagnostic Products Corporation.

\section{Clinical Measures}

In all patients, WHR and BMI (BMI = weight $/$ height squared $[\mathrm{kg} / \mathrm{m} 2]$ ) were determined. In addition, clinical assessment of hirsutism was determined using the Ferriman-Gallwey Scoring System (F/G score) (Gallwey \&
Gallwey, 1961). Nine body sites (the upper lip, chin, chest, upper back, lower back, upper abdomen, lower abdomen, arm, and thigh) are graded from 0 through 4 , with $0=$ no terminal hair to $4=$ severe hirsutism. Scores can range from 0 to 36 . The normal F/G score for women is zero, a score between 1 and 7 would be considered abnormal female body hair growth, and a score of 8 or above is considered positive for hirsutism (van Hooff et al., 2000).

\section{N long-term physical health consequences of PCOS been neglected, the emotional impact of PCOS has been ignored.}

\section{Instrument}

The PCOSQ (Cronin et al., 1998) was administered to 128 women with PCOS. Each of the 26 items are rated on a 7 -point scale, in which optimal functioning is represented by a 7 and a score of 1 would indicate the poorest level of functioning. For example, one item from the hair subscale asked, "To what extent has the following issue been a problem for you: embarrassment about excessive body hair?" The lower the score, the greater the concern and the more adverse the impact on the woman's perceived health-related quality of life (QOL). The time frame for most items was specified as "during the last two weeks," whereas a few items, including headaches and abdominal bloating, referred to the time frame "in relation to your last menstruation." Because the participants were studied during a current menstrual cycle, the time frame for these items would have been the previous week.

\section{Results}

During the 7-month enrollment, 179 women were invited to participate; 175 women consented and were enrolled, and 158 women completed surveys. The mean age of this sample was 30.4 years $(S D \pm 5.5)$. The majority of women were White $(96.9 \%, n=124)$ and married $(78.1 \%, n=100)$, and most had some education beyond high school $(66.4 \%, n=82)$, including either college or trade school. Most women were employed full time $(75.8 \%, n=97)$.

Tables 1, 2, and 3 present the clinical features of the study sample. The majority of women met the criteria for obesity (see Table 1 ), with more than $74 \%$ of the sample exhibiting a BMI greater than 30 . 
TABLE 1

Obesity Characteristics of PCOS Participants

\begin{tabular}{|lcc|}
\hline Obesity Variable & Percent & $\mathrm{n}$ \\
\hline BMI range & & \\
$<18.5-24.9$ & 17.6 & 11 \\
$25.0-29.9$ & 62.5 & 22 \\
$30.0-44.9$ & $\frac{11.7}{100.0}$ & $\frac{15}{128}$ \\
$>45.0$ & & 80 \\
& \\
Note. PCOS = polycystic ovary syndrome; BMI = body mass index.
\end{tabular}

TABLE 2

Fertility Characteristics of PCOS Participants

\begin{tabular}{|lrc|}
\hline Fertility Variable & Percent & $\mathrm{n}$ \\
\hline Reproductive history & 54.7 & 70 \\
Never pregnant & 11.7 & 15 \\
Has been pregnant: no births, all losses & 9.4 & 12 \\
Has been pregnant: some births, some losses & $\underline{12.2}$ & $\underline{31}$ \\
Has been pregnant: all births, no losses & 100.0 & 128 \\
& & \\
Note. PCOS = polycystic ovary syndrome. & & \\
\hline
\end{tabular}

\section{TABLE 3}

\section{Hyperandrogenism Characteristics of PCOS Participants}

\begin{tabular}{|c|c|c|c|c|}
\hline Hyperandrogenism Variable & Mean (SE) & Range & $\%$ Participants & $\mathbf{n}$ \\
\hline Total testosterone $\mathrm{ng} / \mathrm{ml}$ & $82.90(4.25)$ & & & \\
\hline Normal TT & $66.66(3.21)$ & $20-129$ & 83 & 106 \\
\hline Hyperandrogenism TT & $131.09(5.92)$ & $131-238$ & 17 & 22 \\
\hline Free androgen index & $14.16(2.26)$ & & & \\
\hline Normal FAI & $4.69(0.33)$ & $0.46-9.69$ & 54 & 69 \\
\hline Hyperandrogenism FAI & $25.25(4.50)$ & $10.10-242$ & 46 & 59 \\
\hline Ferriman-Gallwey (F/G) score & $12.77(0.76)$ & & & \\
\hline Normal F/G & $3.16(0.42)$ & $0-7$ & 31 & 40 \\
\hline Hyperandrogenism F/G & $17.14(0.69)$ & $8-34$ & 69 & 88 \\
\hline Waist-to-hip ratio & $0.82(.001)$ & & & \\
\hline Normal WHR & $0.79(.004)$ & $0.64-0.85$ & 72 & 89 \\
\hline Hyperandrogenism WHR & $0.90(.007)$ & $0.86-1.03$ & 28 & 34 \\
\hline
\end{tabular}

Reproductive history is summarized in Table 2 as a fertility variable. Two thirds of the sample had never been pregnant nor had successfully carried a pregnancy to term.

Although a large majority of the sample demonstrated TT and WHRs in the normal range, as noted in Table 3, more than two thirds of the women exhibited clinical features of hirsutism, with mean F/G scores for the entire PCOS sample elevated $(M=12.77, S E \pm 0.76)$, whereas mean $F / G$ scores for those with hirsutism (F/G scores $\geq 8$ ) were markedly elevated $(M=17.14, S E \pm 0.69)$.

\section{PCOSQ Subscale Rankings}

Figure 1 presents a summary of the mean scores of the five subscales of the PCOSQ in order of impact from least concern (optimal functioning) to greatest reported concern (poorest functioning), along with a total PCOSQ score calculated from the five subscales. The lower the score, the greater the negative impact on health-related
QOL. In order of importance from least to greatest concern were the scores for (1) body hair, (2) emotions, (3) infertility, (4) menstrual problems, and finally (5) weight, which was the highest health-related QOL concern reported in this sample of 128 women with PCOS. The mean score calculated for the total PCOSQ had greater negative impact than the emotions subscale and was similar to the subscale for infertility.

\section{Reliability of the PCOSQ}

The $\alpha$ reliability coefficient for the total PCOSQ 26item scale was 0.89 . Internal consistency and reliability coefficients for the five subscales in the current study ranged from 0.76 to 0.96 . These findings are summarized in Table 4. One item originally assigned by the developers to the emotions subscale was "How much were you worried about late menstrual period?" This item seemed intuitively better placed in the menstrual subscale, and when 


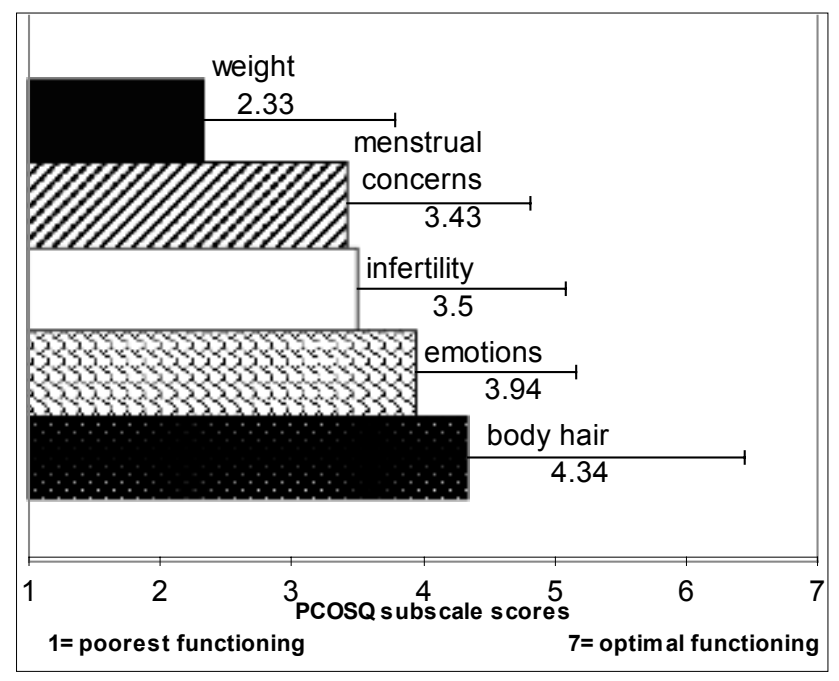

FIGURE 1

Mean scores of five subscales from subjects in current study $(n=128)$ for PCOSQ Quality of Life concerns identified by Cronin et al. (1998). Reported in order of impact on the women from 1 = poorest functioning (lower scores $=$ greater concern) to $7=$ optimal functioning (higher scores $=$ lower concern).

subscale reliability was rerun, moving the item from the emotions to the menstrual subscale significantly improved the $\alpha$ reliability coefficient for both subscales.

\section{Obesity and Quality of Life}

Weight was the greatest concern reported by the PCOS women. Items for the weight subscale included "trouble dealing with weight" and "frustration in losing weight." BMI was determined to be significantly and negatively correlated with the weight subscale of the PCOSQ $(r=$ $-.33, p=.001)$. Simple linear regressions were used to determine whether the degree of obesity (BMI) predicted scores on the PCOSQ. Weight subscale concerns were predicted by BMI $(F=15.581, p=.001)$, such that the higher the BMI (more obese), the lower the scores reported on the PCOSQ weight subscale (indicative of greater weight-related concerns). None of the other subscales (emotion, body hair, infertility, or menstrual problems) were predicted by the degree of obesity as measured by BMI.

\section{Infertility and Quality of Life}

Reproductive history, more specifically the delivery of a viable infant, predicted scores on the infertility concern subscale of the PCOSQ $(F=16.11, p=.001)$. An ANOVA was performed on the PCOSQ infertility subscale using the fertility characteristics (see Table 2). Less than one quarter of the participants had experienced a live birth, and half of the sample was currently undergoing fertility treatment in order to conceive.
TABLE 4

A Comparison of PCOSQ Reliability Scores

With One Subscale Item Exchanged With Those

Originally Assigned by Cronin et al. (1998)

\begin{tabular}{|c|c|c|}
\hline $\begin{array}{l}\text { PCOSQ Domain As } \\
\text { Subscales }\end{array}$ & $\begin{array}{l}\text { Alpha of Subscales } \\
\text { as Originally } \\
\text { Assigned by Cronin } \\
\text { et al. (1998) }\end{array}$ & $\begin{array}{l}\text { Alpha of Subscales } \\
\text { With One Item } \\
\text { Exchanged }\end{array}$ \\
\hline Body hair & 0.96 & 0.96 \\
\hline Weight & 0.93 & 0.93 \\
\hline Infertility & 0.76 & 0.76 \\
\hline Menstrual problems* & $* 0.60$ (4-item) & 0.81 (5-item) \\
\hline Emotions* & 0.56 (8-item) & 0.86 (7-item) \\
\hline Total PCOSQ scale & 0.89 & 0.89 \\
\hline
\end{tabular}

Note PCOSQ = Health-Related Quality of Life Questionnaire.

"Indicates the subscales in which items were exchanged from the original scale.

Women with PCOS who had been pregnant but had experienced only pregnancy losses (no viable infants) reported the lowest scores (greatest concerns) on the infertility subscale $(M=2.57, S E \pm 0.32)$. This exceeded scores of infertility participants unsuccessful in establishing a pregnancy $(M=2.99, S E \pm 0.16)$. These two groups of women had scores that were significantly lower $(F=$ $2.4, p=.001$ ) for the PCOSQ infertility subscale (indicating poorer functioning) than the other two groups of fertile women who had given birth to at least one viable infant. There were no significant differences noted between any of the four fertility groups on the subscales of emotions, body hair, weight, or menstrual problems.

\section{Hyperandrogenism and Quality of Life}

Although the lowest reported subscale of concern in the overall PCOSQ was body hair, this subscale was negatively correlated with the F/G score $(r=-.63, p=.001)$. The higher the F/G score, the lower the reported body hair score, which was indicative of poorer functioning.

The F/G score was also found to be negatively correlated with the emotions subscale scores $(r=-.23, p=$ .001) of the PCOSQ. Hirsute women reported higher scores on the PCOSQ for items in the emotions subscale, including feeling moody, worried, depressed, and having low self-esteem as a result of having PCOS. When women were asked to indicate the impact of emotional symptoms "as a result of your PCOS condition," hirsutism negatively influenced their emotion score. Despite this, the emotion subscale was next to the lowest rated concern on the PCOSQ.

Hyperandrogenism characteristics were correlated with lower scores (poorer emotional health) on two of the 
five PCOSQ subscales. The F/G score negatively impacted both the body hair concerns and emotions subscales. None of the androgen variables was significantly correlated with the subscales for menstrual symptoms, weight, or infertility problems.

\section{Discussion}

This study is one of the first to systematically evaluate the influence of the major clinical features of PCOS on QOL in patients. The PCOSQ instrument correlated well with study variables that addressed specific PCOS symptoms. Not surprisingly, the more adverse the clinical feature (BMI, fertility loss, androgenism), the greater the impact on QOL.

\section{Obesity}

Body dissatisfaction related to real or perceived fatness is widely reported among women in North America (Kolotkin, Head, Hamilton, \& Tse, 1995) and is more common in White women of all sizes (Wilfley, Schreiber, Pike, Striegel-Moore, \& Wright, 1996). PCOS women reported weight as the concern with the greatest impact on their health-related QOL. The current study demonstrated that the higher the participant's BMI, the lower the scores (greater concerns) reported for the weight subscale, supporting the validity of this subscale. The clinical characteristics of the sample may explain why the weight domain was reported to have had the most negative influence on health-related QOL because the majority of participants were obese $(73.4 \%, n=94)$, with a BMI $\geq 30$.

\section{Infertility}

Infertility problems ranked third highest of concern on the PCOSQ (including sadness and worry about not having children and feeling out of control). As noted earlier, more than half the women in the study had never been pregnant. When asked about their current fertility intent, just over half of the study participants reported current plans to attempt pregnancy. The high percentage of women attempting pregnancy and those with previous loss would certainly contribute to infertility concerns ranking third in importance on the PCOSQ.

Current study findings are in general agreement with an earlier study by Orenstein, Raskind, Wyllie, Raskind, and Soules (1986) of psychological consequences in infertile PCOS women compared to infertile women with tubal disease (IWTD) and fertile controls. Orenstein et al. (1986) found infertile PCOS women had higher mean scores for depression than both IWTD and controls. The authors concluded that the high prevalence of negative psychological consequences and somatic complaints reported by infertile PCOS women was unrelated to infertility alone because psychological status was similar among controls and IWTD. However, the study concluded infertility was not responsible for the negative psychological consequences and somatic complaints reported by the PCOS women. Orenstein et al. (1986) reported PCOS distress levels well above norms, but they did not include PCOS women who were not currently attempting to conceive, which was the group reporting the highest levels of emotional distress in the current study (McCook, Reame, \& Thatcher, 2001).

\section{Hyperandrogenism}

Although body hair received the lowest reported area of concern, it was significantly correlated with both the F/G score and the emotions subscale on the PCOSQ. Hirsute women reported higher concerns on the PCOSQ for items in the emotions domain, including feeling moody, worried, depressed, and having low self-esteem as a result of having PCOS.

Sonino, Fava, Mani, Belluardo, and Boscaro (1993) studied nonobese women with hirsutism, including women with PCOS and matched controls using three questionnaires regarding psychosocial problems, psychological distress, and social phobia. When compared to nonhirsute norms, hirsute women reported higher psychological distress and more interpersonal fears, suggesting a compromised QOL (Sonino et al., 1993). Fears reported in the hirsute women were categorized as "social phobia" or anxiety-evoking situations, such as meeting strangers, attending parties, shopping, and mixing at work. However, there were no significant differences reported for depression, somatic symptoms, anger-hostility, or cognitive symptoms between the nonobese hirsute women and controls. These findings suggested the presence of hirsutism did influence those emotions related to social or anxiety-provoking situations but did not influence symptoms such as depression and others not attributed to social phobia.

Shulman, Derogatis, Spielvogel, Miller, and Rose (1992), who investigated the psychopathological aspects

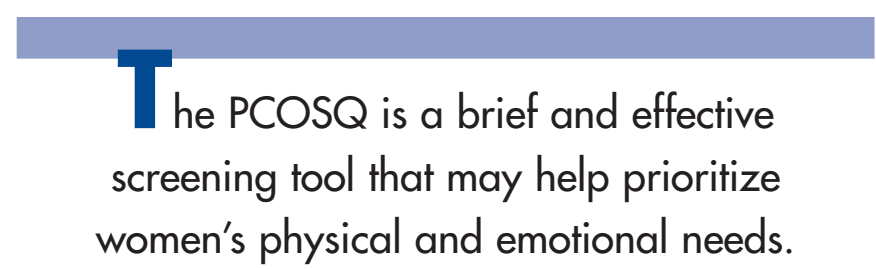

of facial hirsutism, reported significantly elevated scores for negative mood and affect, which was unrelated to the degree of hirsutism and total testosterone. The F/G score was the only hyperandrogenism variable in the current study to significantly influence the body hair subscale of 
American Infertility Association

Center for Applied Reproductive Science

OBGYN.net

PCO Strategies

Polycystic Ovarian Syndrome Association

(PCOSupport)

Note. PCOS = polycystic ovary syndrome. http://www.americaninfertility.org/pcos/pcos_center

http://www.info@ivf-et.com

http://www.obgyn.net

http://www.pcostrategies.org/

http://www.pcosupport.org/ the PCOSQ. PCOS women with elevated F/G scores (marked hirsutism) reported greater hair concerns, as well as more emotional concerns, that is, "feeling moody, worried, depressed, and having low self-esteem."

\section{Conclusions}

The results of this study indicate that women with PCOS have the greatest concern in the area of weight, followed by menstrual problems and infertility. These concerns are directly reflected in their objective life experiences. Women with PCOS clearly need education and support regarding the effect of their condition on their QOL.

Consumer Web sites for patients are a phenomenon that has come of age. There are numerous PCOS information sites and support groups available on the Web. These sites reach thousands of women who feel alone and isolated with their symptoms. One focus of PCOS Web sites is to provide education and emotional support on a variety of topics including depression, infertility treatment, diabetes, weight management, herbal remedies, and medication therapy, including dealing with side effects. For example, at the OBGYN.net PCOS Pavilion Web site (listed in Table 5), a cyber quilt was developed to depict the global implications of PCOS and to serve as a "testament of the need for proper education to assist in a timely diagnosis of this life-altering event" (http://www. obgyn.net). Currently, the cyber quilt includes more than 4,800 individual women's stories of their struggle with the symptoms of PCOS, representing more than 42,000 years of suffering prior to the diagnosis of PCOS.

\section{Limitations}

The use of a relatively homogeneous sample of White, married women attending a private reproductive endocrinology practice in central Appalachia may limit generalization of the findings to the entire PCOS population. Patient populations undergoing or seeking medical treatment are known to differ on a number of sociocultu- ral and psychological parameters compared to community samples. Nelson, Palmer, Pedersen, and Miles (1999) reported depression, anxiety, and impaired self-esteem in obese adults who seek treatment for a variety of health conditions, and this may be the case for these PCOS women as well.

Another potential study limitation was the enthusiasm with which participants agreed to participate in the investigation. Participation agreement was $97.2 \%$, with very few refusals (5 in 179). Not unlike women attending support groups, women seeking treatment for PCOS may be proactive in pursuing care, as evidenced by locating a reproductive endocrinologist with expertise in recognizing PCOS, accessing transportation, and in some cases, traveling hundreds of miles to seek care. Several women expressed relief during the recruitment phase, that someone was interested in their emotional well-being. Thus, women who volunteered to be in the study may be different from other women with PCOS, who may be "suffering in silence," thereby influencing study outcomes.

The absence in this study of non-PCOS patient controls matched on the various clinical features prevented the assessment of their individual and collective contributions as overriding influences on QOL, separate from the diagnosis of PCOS. Control participants for comparison with women with PCOS are very difficult to identify in advance of screening, owing to its high prevalence and diagnostic difficulties discussed above. Comparison studies are also needed with healthy ovulatory women to better define the degree of impaired QOL resulting from PCOS.

\section{Nursing Implications}

The importance of including health-related QOL instruments to evaluate treatment from the patient's perspective and to investigate clinical interventions over time has been established (Colwell et al., 1998; Mant \& Fowler, 1990; Streiner, 1995). The PCOSQ is a brief screening tool nurses and other health care providers can use to help prioritize health-related concerns from the 
patient's prospective. In this way, emotional concerns can be evaluated and prioritized along with physical concerns to provide thorough patient care.

A team approach is necessary to provide comprehensive care, including nutrition, exercise physiology, psychological counseling, and emotional support for the complex management of PCOS. Health care providers must be educated in the assessment, diagnosis, and management of women with PCOS, which is largely underdiagnosed and undertreated.

The sooner a diagnosis of PCOS is reached, the sooner a thorough treatment plan can be initiated. Care includes correcting underlying endocrine imbalances, nutritional counseling, treating skin and excess hair concerns, pursuing fertility treatment if desired, and very important, the provision of psychosocial screening and support.

Nursing needs to recognize the impact of this disorder on women's QOL and identify approaches to support these women. One approach to providing support to women with PCOS is through Web sites developed for that purpose. Women with PCOS are accessing information through the Internet and support groups and are repeatedly relaying concern and frustration that health care providers lack the knowledge and understanding to answer their questions regarding nutrition, emotional consequences (depression, moodiness), medication therapy, and long-term morbidity issues. Health care providers need to be informed about PCOS so they can provide information to their few but increasingly well-informed consumers.

\section{Acknowledgment}

Supported in part by East Tennessee State University College of Nursing, the University of Michigan School of Nursing, the Center for Applied Reproductive Science, and Diagnostics Products Corporation.

\section{REFERENCES}

Cecchine, R. (2001). Scrambled: A journey through polycystic ovarian syndrome. Boston: Fanlight Productions. Available from http://www.scrambledthewebsite.com

Colwell, H., Mathias, S., Pasta, D., Henning, J., \& Steege, J. (1998). A health-related quality of life instrument for symptomatic patients with endometriosis: A validation study. American Journal of Obstetrics and Gynecology, 179, 47-55.

Cronin, L., Guyatt, G., Griffith, L., Wong, E., Azziz, R., Futterweit, W., et al. (1998). Development of a health-related quality-of-life questionnaire (PCOSQ) for women with polycystic ovary syndrome (PCOS). Journal of Clinical Endocrinology and Metabolism, 83(6), 1976-1987.
Gallwey, D., \& Gallwey, J. (1961). Clinical assessment of body hair growth in women. Journal of Clinical Endocrinology and Metabolism, 21, 1440-1447.

Jones, G., Kennedy, S., \& Jenkinson, C. (2002). Health-related quality of life measurement in women with common benign gynecologic conditions: A systemic review. American Journal of Obstetrics and Gynecology, 187(2), 501511.

Kolotkin, R. L., Head, S., Hamilton, M., \& Tse, C. K. (1995). Assessing impact of weight on quality of life. Obesity Research, 3(1), 49-56.

Mant, D., \& Fowler, G. (1990). Mass screening: Theory and ethics. British Medical Journal, 300, 916-918.

McCook, J. G. (2002). The influence of hyperandrogenism, obesity and infertility on the psychosocial health and wellbeing of women with polycystic ovary syndrome. Unpublished doctoral dissertation, University of Michigan.

McCook, J. G., Reame, N. E., \& Thatcher, S. S. (2001). The influence of obesity, androgenism, and fertility status on the psychological health and well-being of women with polycystic ovary syndrome. Abstracts of the Scientific Oral Sessions of the American Society for Reproductive Medicine. Birmingham, AL: American Society for Reproductive Medicine.

Nelson, T. L., Palmer, R. F., Pedersen, N. L., \& Miles, T. P. (1999). Psychological and behavioral predictors of body fat distribution: Age and gender effects. Obesity Research, 7(2), 199-207.

Orenstein, H., Raskind, M. A., Wyllie, D., Raskind, W. H., \& Soules, M. R. (1986). Polysymptomatic complaints and Briquet's syndrome in polycystic ovary disease. American Journal of Psychiatry, 143(6), 768-771.

Shulman, L. H., Derogatis, L., Spielvogel, R., Miller, J. L., \& Rose, L. I. (1992). Serum androgens and depression in women with facial hirsutism. Journal of the American Academy of Dermatology, 27(2 Pt. 1), 178-181.

Sonino, N., Fava, G. A., Mani, E., Belluardo, P., \& Boscaro, M. (1993). Quality of life of hirsute women. Postgraduate Medical Journal, 69(809), 186-189.

Streiner, D. (1995). Health measurement scales: A practical guide to their development and use. Oxford, UK: Oxford University Press.

Thatcher, S. S. (2000). Polycystic ovary syndrome: The hidden epidemic. Indianapolis, IN: Perspectives Press.

van Hooff, M., Voorhorst, F., Kaptein, M., Hirasing, R., Koppenaal, C., \& Schoemaker, J. (2000). Polycystic ovaries in adolescents and the relationship with menstrual cycle patterns, luteinizing hormone, androgens, and insulin. Fertility and Sterility, 74(1), 49-58.

Vankrieken, L. (1997). Testosterone and the free androgen index (ZB158 ed.). Los Angeles: Diagnostics Products.

Wilfley, D. E., Schreiber, G. B., Pike, K. M., Striegel-Moore, R. H., \& Wright, D.J.R.J. (1996). Eating disturbance and body image: A comparison of a community sample of adult black and white women. International Journal of Eating Disorders, 20(4), 377-387. 
Judy Griffin McCook, PhD, RN, BC, is an associate professor, Family and Community Nursing, East Tennessee State University College of Nursing, Johnson City. She is also at the Center for Applied Reproductive Science, Johnson City, TN \& Asheville, NC.

Nancy E. Reame, PhD, MSN, FAAN, is the Rhetaugh Graves Dumas Professor of Nursing, University of Michigan School of Nursing, Ann Arbor, and a research scientist in the Reproduc- tive Science Program, Department of Obstetrics and Gynecology, University of Michigan, Ann Arbor.

Samuel S. Thatcher, MD, PhD, is director of the Center for Applied Reproductive Science, Johnson City, TN, \& Asheville, NC.

Address for correspondence: Judy Griffin McCook, PhD, RN, BC, East Tennessee State University College of Nursing, Johnson City, TN. E-mail: mccook@etsu.edu. 